\title{
Salvianolic acid A protects against myocardial ischemia/reperfusion injury by reducing platelet activation and inflammation
}

\author{
XIAOLING YUAN* ${ }^{*}$ YIJIA XIANG* , NING ZHU, XUYONG ZHAO, SHIYONG YE, \\ PENG ZHONG and CHUNLAI ZENG
}

Department of Cardiology, The Fifth Affiliated Hospital of Wenzhou Medical University, Lishui, Zhejiang 323000, P.R. China

Received April 13, 2016; Accepted March 17, 2017

DOI: $10.3892 /$ etm.2017.4619

\begin{abstract}
The aim of the present study was to investigate the protective effect of salvianolic acid A (SAA) on myocardial ischemia/reperfusion injury in rats. SAA $(10 \mathrm{mg} / \mathrm{kg})$ or Tirofiban $(60 \mu \mathrm{g} / \mathrm{kg})$ was administered to rats by jugular vein injection $10 \mathrm{~min}$ before the initiation of reperfusion. After $3 \mathrm{~h}$ of reperfusion, platelet aggregation was measured using an aggregometer and levels of nitric oxide (NO) were detected using an ultraviolet spectrophotometer. Serum levels of cardiac troponin $\mathrm{T}$ (cTnT), creatine kinase isoenzyme MB (CK-MB), $p$-selectin, interleukin-1 $\beta$ (IL-1 $\beta$ ) and tumor necrosis factor- $\alpha$ (TNF- $\alpha$ ) were also measured 3 and $24 \mathrm{~h}$ after reperfusion. Furthermore, morphology of the ischemic myocardium was histopathologically analyzed by hematoxylin and eosin staining, and the infarct area was evaluated by Evans blue and triphenyltetrazolium chloride staining. In rats subjected to reperfusion, it was observed that pretreatment with SAA significantly increased the survival rate $(\mathrm{P}<0.05)$, and that increased survival rate was due to a significant decrease in infarct size, as evidenced by significantly reduced serum levels of cTnT and CK-MB $(\mathrm{P}<0.05)$. In addition, decreases in infarct size occurred through the inhibition of platelet aggregation and inflammation associated with reperfusion-induced myocardial cell damage, as indicated by reduced serum levels of p-selectin, TNF- $\alpha$, IL-1 $\beta$ and NO. In conclusion, SAA was protective against myocardial ischemia/reperfusion injury in rats by serving antiplatelet and anti-inflammation roles.
\end{abstract}

Correspondence to: Dr Chunlai Zeng, Department of Cardiology, The Fifth Affiliated Hospital of Wenzhou Medical University, 289 Kuocang Road, Lishui, Zhejiang 323000, P.R. China

E-mail: zengchunlai@medmail.com.cn

*Contributed equally

Key words: salvianolic acid A, myocardial ischemia reperfusion, infarct area, platelet aggregation rate, inflammation

\section{Introduction}

Acute myocardial infarction (AMI) is a major public health problem. In 2010, age-standardized AMI incidence in all ages was 195.3 per 100,000 in males and 115.0 in females (1). In Europe, $\sim 1.8$ million individuals succumb to coronary heart disease each year (2). Reperfusion of the ischemic myocardium is necessary to salvage tissue and limit cardiomyocyte death. While reperfusion is beneficial, it also induces new pathophysiological changes through the release of oxygen free radicals, cytokines and other pro-inflammatory factors that initiate an inflammatory cascade. This inflammation typically enhances damage to myocytes and induces myocardial dysfunction (3-5). Platelets serve key roles in myocardial reperfusion injury, including platelet adhesion, aggregation, activation and inhibiting the platelet activation, which reduces myocardial infarct size (6). Furthermore, they induce new thrombus formation and microembolization at sites of atherosclerotic plaques in coronary arteries, and facilitate interactions between the endothelium and leukocytes, consequently prompting microcirculation disturbance and inflammation (7-9). Suppression of platelet activation using Tirofiban to inhibit glycoprotein IIb/IIIa may alleviate ischemia-reperfusion (I/R) injury $(10,11)$.

The Traditional Chinese Medicine Salvia miltiorrhiza is widely used in the treatment of coronary artery disease and cerebrovascular diseases and as a remedy to improve microcirculation $(12,13)$. Salvianolic acid A (SAA), as a watersoluble polyphenolic compound, is a primary constituent of Salvia miltiorrhiza and is extracted from Salvia miltiorrhiza Bunge (14). A previous study by our group revealed that SAA dose-dependently inhibited platelet aggregation induced by adenosine diphosphate (ADP), thrombin, collagen and U46619, and inhibited arterial thrombosis via the inhibition of phosphoinositide 3-kinase in vitro (15). In the present study, the cardioprotective effects of SAA against platelet activation and inflammation during myocardial $\mathrm{I} / \mathrm{R}$ injury were investigated by ligating the left anterior descending branch of coronary artery. Results indicated that the cardioprotective effects of SAA were comparable to those of the positive control agent, Tirofiban. 


\section{Materials and methods}

Animals. A total of 150, male Sprague-Dawley rats (aged 6-8 weeks and weighing 250-300 g) were purchased from the Zhejiang Laboratory Animal Centre, China [certificate no. SCXL (Zhe) 2008-0033]. Animals were acclimated for at least 1 week at room temperature $\left(18-25^{\circ} \mathrm{C}\right), 55 \pm 5 \%$ humidity and a 12-h light/dark cycle. The animals were given free access to a standard diet and tap water. All experimental procedures were approved by the Ethics Review of Animal Use Application of Fifth Affiliated Hospital of Wenzhou Medical University (Zhejiang, China).

Animal groups and drug pretreatment. All animals were randomly assigned to one of four groups. Each group was divided into $\mathrm{n} 1$ and $\mathrm{n} 2$ subgroups and exposed to reperfusion for 3 and $24 \mathrm{~h}$, respectively. The model control group (I/R; $\mathrm{n} 1=10$, $\mathrm{n} 2=30$ ) underwent $\mathrm{I} / \mathrm{R}$ and received an intravenous administration of glucose $(5 \%)$, while the SAA group (I/R + SAA, $\mathrm{n} 1=10, \mathrm{n} 2=30$ ) underwent $\mathrm{I} / \mathrm{R}$ and received an intravenous administration of SAA $(10 \mathrm{mg} / \mathrm{kg}$; Zhengda Qingchunbao Pharmaceutical Co., Ltd., Zhejiang, China) dissolved in 5\% glucose. The Tirofiban group (I/R + Tirofiban, $\mathrm{n} 1=10, \mathrm{n} 2=30$ ) underwent $\mathrm{I} / \mathrm{R}$ and received an intravenous administration of Tirofiban $(60 \mu \mathrm{g} / \mathrm{kg}$; Wuhan Grand Pharmaceutical Co., Ltd., Wuhan, China) dissolved in 5\% glucose. The sham group (sham; $\mathrm{n} 1=10, \mathrm{n} 2=20$ ) received an intravenous administration of glucose $(5 \%)$ alone. All drugs were administered intravenously $10 \mathrm{~min}$ before reperfusion was initiated.

Myocardial I/R model protocol. Rats were anesthetized with an intraperitoneal injection of $4 \%$ chloral hydrate $(400 \mathrm{mg} / \mathrm{kg})$. Following intubation, a left thoracotomy was performed. The left anterior descending coronary artery (LAD) was surgically occluded for $45 \mathrm{~min}$ through ligation of a silk suture, and coronary occlusion was confirmed by elevation of the ST segment $(>0.1 \mathrm{mV})$ on an electrocardiogram (MedLab-U/4C50; Nanjing Medease Science and Technology Co., Ltd., Nanjing, China). Reperfusion of the coronary artery was initiated by release of the ligation tie. Following the procedure, the chest was closed and rats in each group; $\mathrm{n} 1$ and $\mathrm{n} 2$ were monitored in the animal facility for 3 and $24 \mathrm{~h}$, respectively, anesthetized with an intraperitoneal injection of $4 \%$ chloral hydrate ( $400 \mathrm{mg} / \mathrm{kg}$ ), prior to sacrifice with $10 \%$ potassium chloride solution $(400 \mathrm{mg} / \mathrm{kg} ; 1-2 \mathrm{ml})$ administered to the inferior vena cava. All groups underwent the same surgical procedure, though the LAD suture was not tied for rats in the sham group.

Measurement of myocardial infarct size. Myocardial infarct size was evaluated by Evans blue and 2,3,5-triphenyl-2H-tetrazolium chloride (TTC) staining (both from Sigma-Aldrich, Inc.; Merck KGaA, Darmstadt, Germany), as described previously (16). Briefly, after $24 \mathrm{~h}$ of reperfusion, rats were sacrificed and the LAD was occluded with a silk suture in the same location as for the I/R procedure. The abdomen was opened and $5 \mathrm{ml}$ of $1 \%$ Evans blue dye was injected into the aorta. The heart was immediately excised and the right ventricle and left and right atria were removed. The left ventricle was transversally cut into $1 \mathrm{~mm}$ thick slices and incubated in $1 \%$ TTC at $37^{\circ} \mathrm{C}$ for $10 \mathrm{~min}$. Evans blue was used to stain the area outside of the risk area (RA), and the area unstained by TTC represented the ischemic area. The infarct area (ischemic area), RA and left ventricle wall size were also measured digitally using Image J software (version 1.38; National Institutes of Health, Bethesda, MA, USA).

Determination of platelet maximum aggregation rate. Blood was collected from the abdominal aorta in an anticoagulant solution containing 3.8\% sodium citrate (Sinopharm Chemical Reagent Co., Ltd., Shanghai, China), 1:9 citrate: whole blood) after $3 \mathrm{~h}$ of reperfusion. The platelet-rich plasma (PRP) fraction was obtained by centrifugation at $174 \mathrm{xg}$ at $25^{\circ} \mathrm{C}$ for $10 \mathrm{~min}$, and the remaining blood was further centrifuged at $1,570 \mathrm{x} \mathrm{g}$ at $25^{\circ} \mathrm{C}$ for $10 \mathrm{~min}$ to prepare the platelet-poor plasma (PPP) fraction. The platelet concentration, according to a previously described method, using 1x20 1 Coulter Isoton ${ }^{\circledR}$ II Dilutent (cat. no. 8546719; Beckman Coulter, Brea, CA, USA) (15), was adjusted to $250 \times 10^{6}$ platelets $/ \mathrm{ml}$ and incubated at room temperature for $30 \mathrm{~min}$ to allow the platelets to clot. The platelet agonist ADP disodium ( $5 \mu \mathrm{M}$ final concentration; Helena Laboratories, Beaumont, TX, USA) was used to stimulate platelet aggregation as a positive control. The level of platelet aggregation was measured using an aggregometer (AggRAM; cat. no. 8JF52001; Helena Laboratories, Beaumont, Texas, USA).

Enzyme-linked immunosorbent assay (ELISA). After 3 and $24 \mathrm{~h}$ of reperfusion, blood samples were collected from the abdominal aorta using $3.8 \%$ sodium citrate as the anticoagulant, and samples were centrifuged at $25^{\circ} \mathrm{C}$ and $3,500 \mathrm{xg}$ for $15 \mathrm{~min}$ to isolate the plasma. Quantikine ELISA kits (96Test; Abcam, Cambridge, UK) were used to measure the plasma levels of creatine kinase isoenzyme MB (CK-MB; cat. no. XF020852B), cardiac troponin T (cTnT; cat. no. XF03363B), p-selectin (cat. no. XF03259B), interleukin-1 $\beta$ (IL-1 $\beta$; cat. no. XF01588B) and tumor necrosis factor- $\alpha$ (TNF- $\alpha$; cat. no. XF01721B), according to the manufacturer's protocol.

Histological analysis by light microscopy. After $24 \mathrm{~h}$ of reperfusion, myocardial samples from the RA were rinsed with PBS $(\mathrm{pH} 7.4)$ and fixed in $4 \%$ paraformaldehyde and $25^{\circ} \mathrm{C}$. After $24 \mathrm{~h}$ fixation, tissues were dehydrated in graded alcohol $(75,85$ and $95 \%$ twice and then $100 \%$ twice) at a temperature of $25^{\circ} \mathrm{C}$, embedded in paraffin and cut into 3-5 $\mu \mathrm{m}$ thin sections. The tissue sections were then stained with hematoxylin and eosin and histologically examined under a light microscope.

Measurement of myocardial nitric oxide (NO) content. The myocardial NO concentration was measured as described previously (17). Briefly, after $3 \mathrm{~h}$ of reperfusion, myocardial samples from the RA were rinsed and homogenized in deionized water $(1: 10 \mathrm{w} / \mathrm{v})$ prior to centrifugation at $3,000 \mathrm{xg}$ at $25^{\circ} \mathrm{C}$ for $5 \mathrm{~min}$. The concentration of $\mathrm{NO}$ in the supernatant was determined using an NO detection kit (Nanjing Jiancheng Bioengineering Institute, Nanjing, China), according to the manufacturer's instructions.

Statistical analysis. All data are presented as the mean \pm standard error of the mean and were analyzed by one-way analysis of variance, followed by a least-significant-difference test for multiple comparisons. Survival rate was analyzed by 
A

Sham
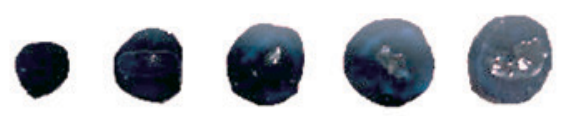

B I/R
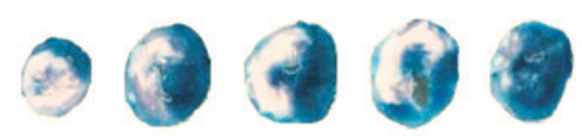

SAA
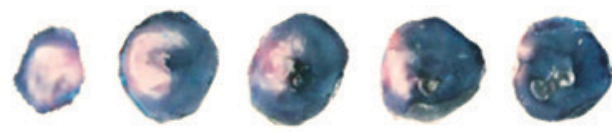

Tirofiban
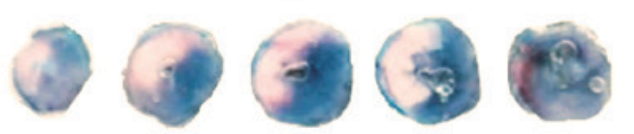
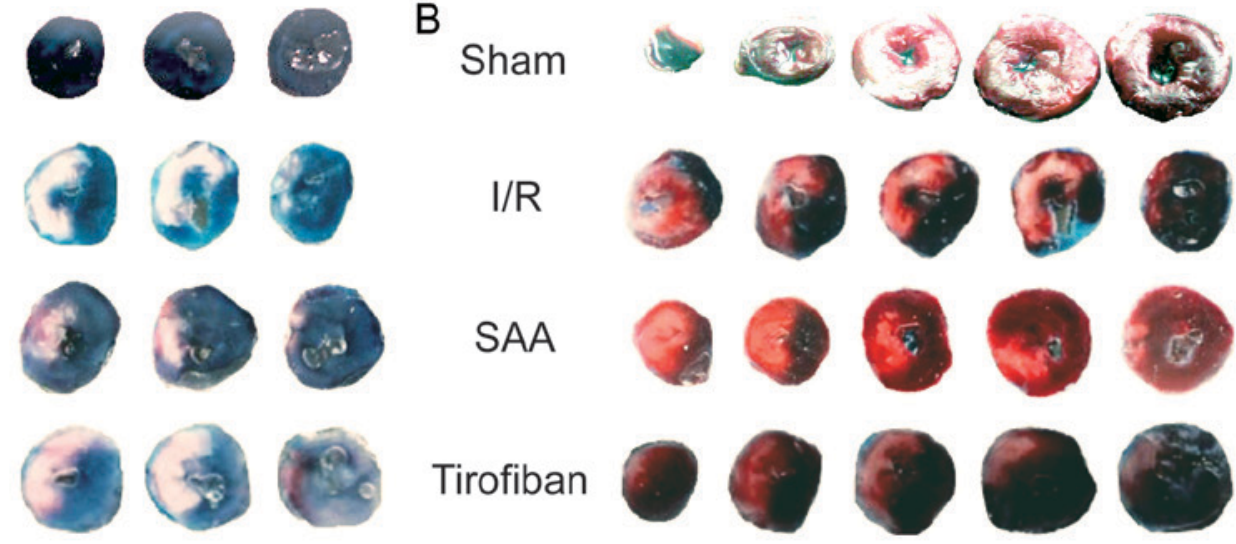

Tirofiban
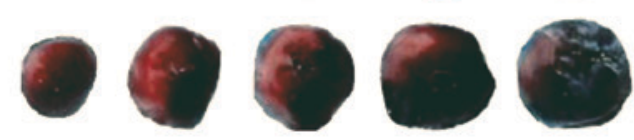

Figure 1. Morphological assessment of left ventricles exposed to I/R. Representative Evans blue staining of the left ventricles in the (A) Sham, I/R, SAA and Tirofiban group was used to determine the RA. Normal myocardium was stained blue and the RA was stained white. Representative tetrazolium chloride staining of the left ventricles in the (B) Sham, I/R, SAA and Tirofiban group was used to determine infarct size. Normal myocardium was stained red and the infarct area was stained pale red and/or pink. I/R, ischemia-reperfusion; SAA, salvianolic acid A; RA, risk area.

Table I. Effect of SAA and Tirofiban on myocardial I/R injury in rats.

\begin{tabular}{lccc}
\hline Group & Dosage & Risk area, $\%$ & Infarct area, $\%$ \\
\hline Sham & - & NA & NA \\
I/R & - & $30 \pm 2$ & $29 \pm 5$ \\
SAA & $10 \mathrm{mg} / \mathrm{kg}$ & $29 \pm 4$ & $19 \pm 11^{\mathrm{a}}$ \\
Tirofiban & $60 \mu \mathrm{g} / \mathrm{kg}$ & $29 \pm 3$ & $19 \pm 4^{\mathrm{a}}$ \\
\hline
\end{tabular}

Values are presented as the mean \pm standard error of the mean. ${ }^{a} \mathrm{P}<0.05$ vs. I/R. SAA, salvianolic acid A; I/R, ischemia-reperfusion; NA, not applicable.

Kaplan-Meier analysis with SPSS, version 19.0 software (IBM Corp., Armonk, NY, USA). P $<0.05$ was determined to represent statistically significant differences following a log-rank test.

\section{Results}

SAA treatment reduces myocardial injury and mortality following myocardial $I / R$. After $24 \mathrm{~h}$ of reperfusion, the RAs of the SAA, Tirofiban and control I/R groups were all of a similar size. The infarct areas were significantly reduced in both the SAA and Tirofiban groups compared to the I/R group (both $\mathrm{P}<0.05$; Table I; Fig. 1). Furthermore, survival rates of rats in the sham, I/R, SAA and Tirofiban groups were 100,61, 82 and $63 \%$, respectively (log-rank test $\mathrm{P}=0.033$ SAA group vs. I/R group; Fig. 2), indicating that rats in the SAA group had an improved survival rate relative to those in the I/R and Tirofiban groups. In addition, after 3 and $24 \mathrm{~h}$ of reperfusion, levels of cTnT and CK-MB in the serum were significantly increased in the $\mathrm{I} / \mathrm{R}$ group, relative to the sham group (both $\mathrm{P}<0.05)$. This effect was significantly reversed by pretreatment with SAA or Tirofiban after $24 \mathrm{~h}$ reperfusion (all $\mathrm{P}<0.05$; Fig. 3).

Under light microscopy, the myocardial samples of the $\mathrm{I} / \mathrm{R}$ group exhibited a disordered myocardial cell arrangement

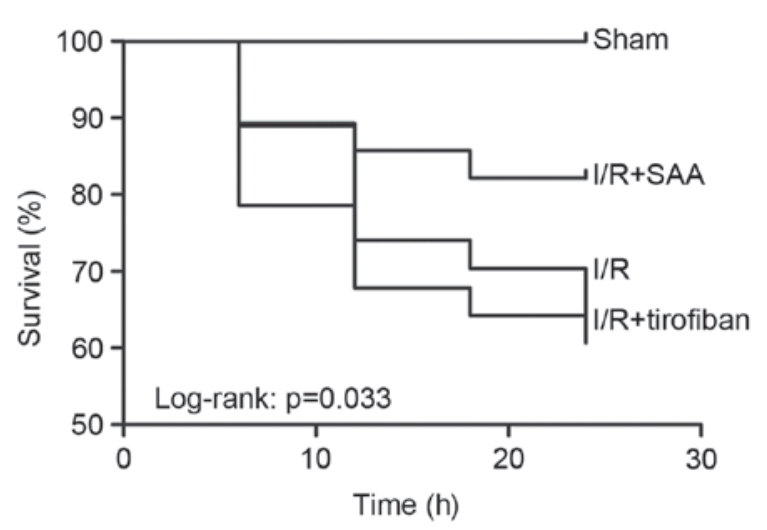

Figure 2. Survival rate $24 \mathrm{~h}$ after myocardial ischemia-reperfusion injury. $\mathrm{I} / \mathrm{R}$, ischemia-reperfusion; SAA, salvianolic acid A.

and robust inflammatory cell infiltration, relative to the Sham group. In turn, myocardial injury and inflammatory exudation were attenuated in both the SAA and Tirofiban groups (Fig. 4).

SAA treatment inhibits platelet aggregation during myocardial $I / R$. After $3 \mathrm{~h}$ of reperfusion, the maximum rate of platelet aggregation in the $\mathrm{I} / \mathrm{R}$ group was similar to that in the sham group $(P=0.195)$. This rate was significantly reduced by pretreatment with SAA or Tirofiban (both $\mathrm{P}<0.05$ vs. I/R group). The platelet aggregation rates of the SAA and Tirofiban groups did not differ significantly ( $\mathrm{P}=0.59$; Fig. 5).

SAA treatment reduces serum levels of p-selectin. After 3 or $24 \mathrm{~h}$ of reperfusion, levels of p-selectin in the serum were significantly increased in the $\mathrm{I} / \mathrm{R}$ group, compared with the sham group $(\mathrm{P}<0.05)$. This effect was significantly reversed by pretreatment with SAA or Tirofiban $(\mathrm{P}<0.05$ vs. I/R). Serum levels of $p$-selectin did not differ significantly between the SAA and Tirofiban groups ( $\mathrm{P}>0.05$; Fig. 6$)$.

SAA treatment reduces serum levels of TNF- $\alpha$ and $I L-1 \beta$. After $24 \mathrm{~h}$ of reperfusion, levels of TNF- $\alpha$ in the serum significantly increased in the I/R group compared to the sham group (both $\mathrm{P}<0.05$ ). In turn, pretreatment with SAA or Tirofiban 

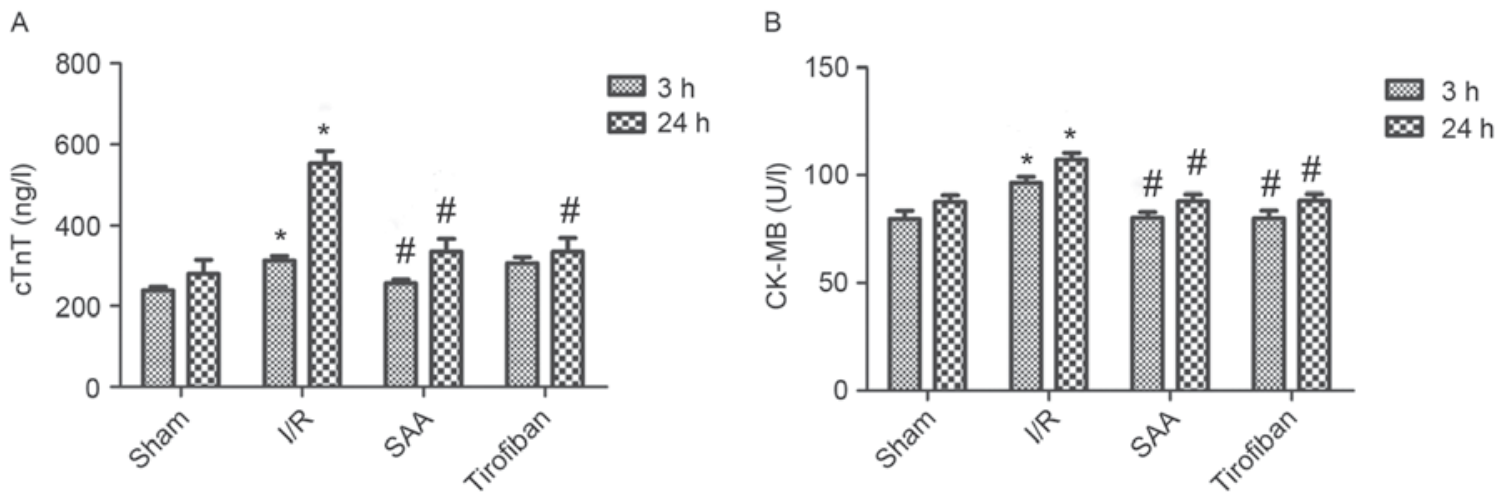

Figure 3. SAA and Tirofiban reduced serum levels of cTnT and CK-MB following myocardial I/R. (A) cTnT and (B) CK-MB activity were quantified by ELISA. Data are presented as the mean \pm standard error of the mean. ${ }^{~} \mathrm{P}<0.05$ vs. sham, ${ }^{\prime} \mathrm{P}<0.05$ vs. I/R. cTnT, cardiac troponin T; CK-MB, creatine kinase isoenzyme $\mathrm{MB} ; \mathrm{I} / \mathrm{R}$, ischemia-reperfusion; SAA, salvianolic acid A.
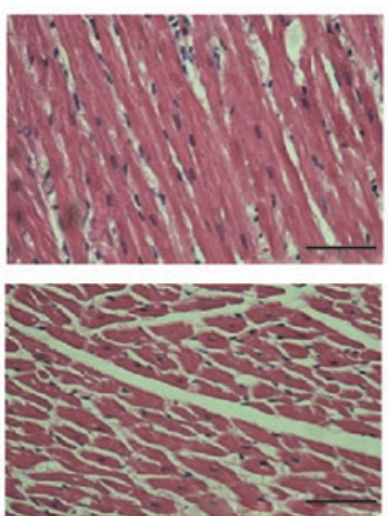

Sham
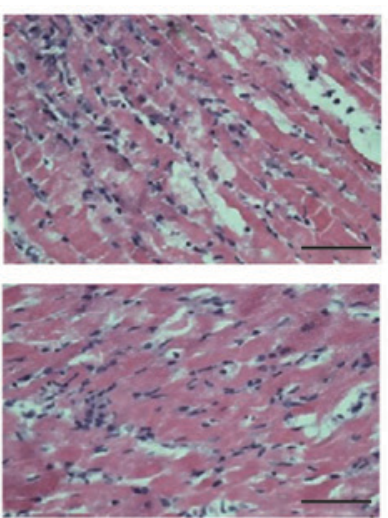

I/R
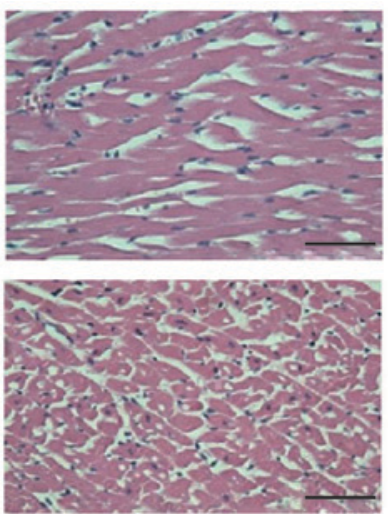

SAA
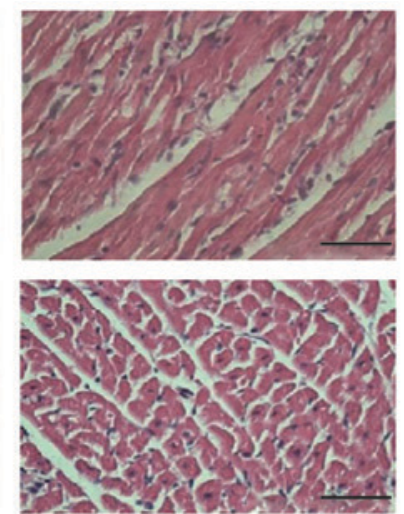

Tirofiban

Figure 4. Cross-sectional and longitudinal sections of hematoxylin and eosin stained myocardial tissue at $3 \mathrm{~h}$ after reperfusion. Magnification, x40. Scale bar, $100 \mu \mathrm{m} . \mathrm{I} / \mathrm{R}$, ischemia-reperfusion; SAA, salvianolic acid A.

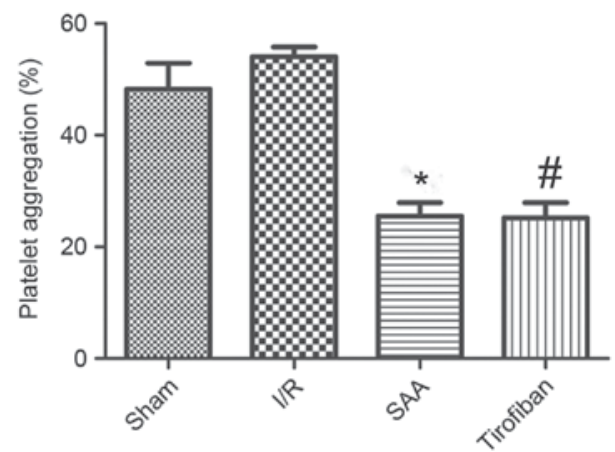

Figure 5. SAA and Tirofiban reduced platelet aggregation induced by ADP stimulation. Platelets were isolated from the blood of sham rats or rats following I/R. Data shown are presented as the mean \pm standard error of the mean. ${ }^{~} \mathrm{P}<0.05 \mathrm{SAA}$ vs. $\mathrm{I} / \mathrm{R}$ group, ${ }^{\text {" }} \mathrm{P}<0.05$ Tirofiban vs. $\mathrm{I} / \mathrm{R}$ group. $\mathrm{SAA}$, salvianolic acid A; ADP, adenosine diphosphate; I/R, ischemia-reperfusion.

significantly reduced serum levels of TNF- $\alpha$ after $24 \mathrm{~h}$ of reperfusion (both $\mathrm{P}<0.05$; Fig. 7A).

Similarly, after 3 or $24 \mathrm{~h}$ of reperfusion, levels of IL-1 $\beta$ in the serum were significantly increased in the I/R group, relative to the sham group (both $\mathrm{P}<0.05$ ). In turn, pretreatment with SAA significantly reduced the serum levels of IL-1 $\beta$ levels (both $\mathrm{P}<0.05$ compared to I/R) such that the serum levels of IL-1 $\beta$ did

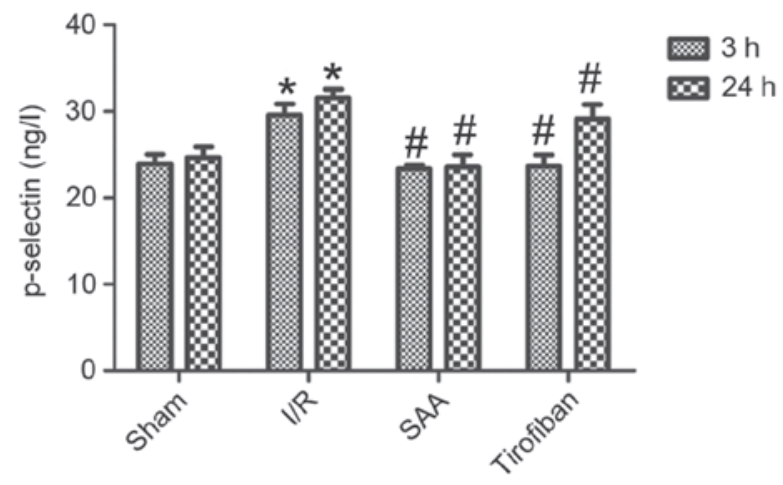

Figure 6. SAA reduced serum levels of p-selectin following myocardial I/R. Data are presented as the mean \pm standard error of the mean. "P<0.05 vs. sham; ${ }^{\#}<0.05$ vs. I/R. SAA, salvianolic acid A; I/R, ischemia-reperfusion.

not differ significantly between the SAA and Tirofiban groups (P>0.05; SAA vs Tirofiban group for 3 or 24 h; Fig. 7B).

SAA treatment increases the NO concentration in myocardial tissue. After $3 \mathrm{~h}$ of reperfusion, concentrations of NO in the RA were similar between the $I / R$ and sham groups $(P>0.05)$. Relative to the I/R the SAA group, but not the Tirofiban group, 
A

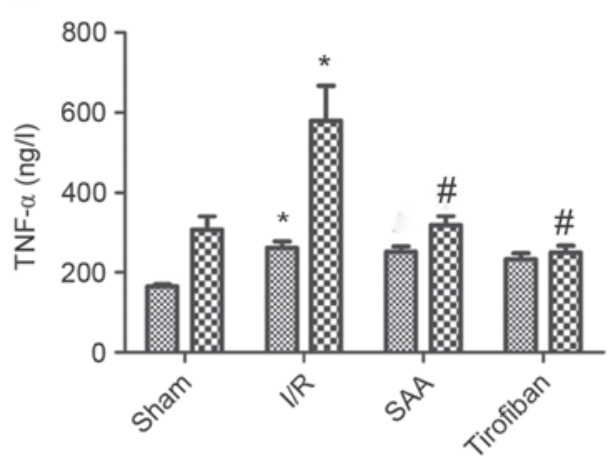

B

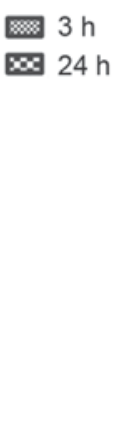

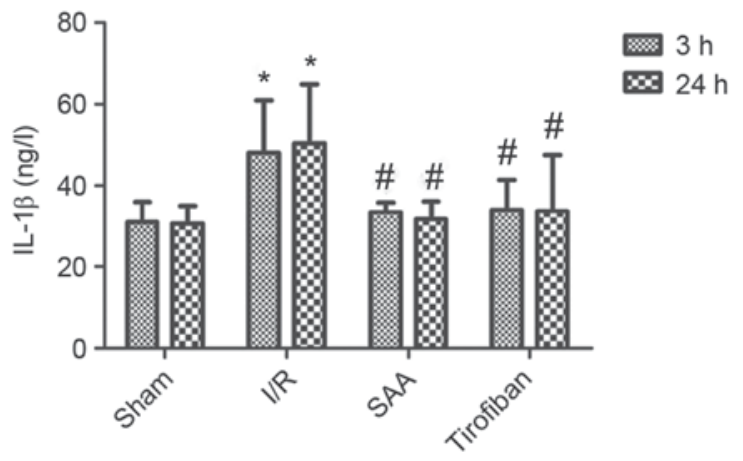

Figure 7. SAA and Tirofiban reduced serum levels of IL- $\beta$ and TNF- $\alpha$ following myocardial I/R. (A) TNF- $\alpha$ and (B) IL-1 $\beta$ were quantified by ELISA. Data are presented as the mean \pm standard error of the mean. ${ }^{*} \mathrm{P}<0.05$ vs. sham; ${ }^{*} \mathrm{P}<0.05$ vs. I/R. SAA, salvianolic acid A; I/R, ischemia-reperfusion; IL-1 $\beta$, interleukin-1 $\beta$; TNF- $\alpha$, tumor necrosis factor- $\alpha$.

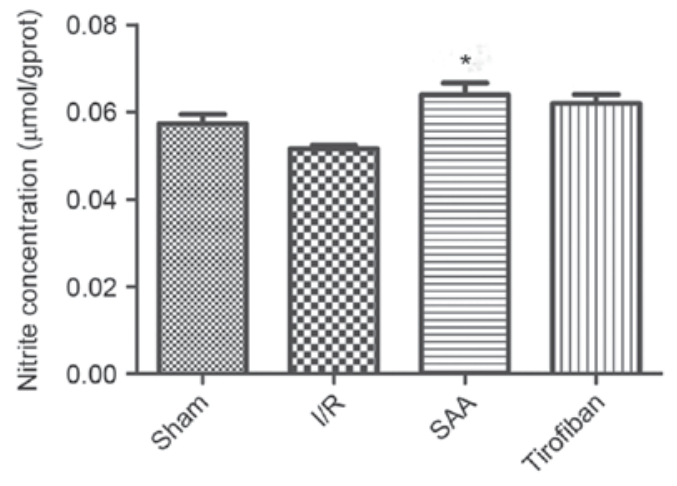

Figure 8. SAA treatment increased NO concentration in the left ventricle following myocardial I/R. Data are presented as the mean \pm standard error of the mean. ${ }^{*} \mathrm{P}<0.05$ vs. I/R. SAA, salvianolic acid A; I/R, ischemia-reperfusion.

exhibited significantly increased concentrations of NO in the RA (P<0.05; Fig. 8).

\section{Discussion}

The present study demonstrated that pretreatment with SAA significantly ameliorated cardiac injury and improved the survival rate of rats exposed to I/R. SAA reduced serum levels of cTnT and CK-MB and myocardial infarct size. Furthermore, SAA inhibited ADP-induced platelet aggregation, decreased IL-1 $\beta$ and TNF- $\alpha$ activity, reduced swelling of ischemic myocardial cells and inflammatory cell infiltration, and increased NO synthesis in the area at risk. These observations indicate that SAA may be a potential therapeutic agent in the treatment of myocardial I/R injury.

Platelets serve critical roles in I/R injury. Following reperfusion, platelet contact with subendothelial collagen stimulates platelet adhesion and activation (18). Activated platelets generate a variety of factors that induce a feed forward loop to reinforce the activation process. For instance, thromboxane A2 enhances platelet activation and adhesion, while ADP and thrombin recruit circulating platelets and promote platelet aggregation. Concomitant with these events, tissue factor XIII is activated to promote the formation of clots (both large thrombi and small platelet microthrombi). In the present study, SAA exhibited strong antiplatelet activity, as observed in an in vitro platelet aggregation assay. The in vitro experiments of the present study demonstrated that treatment with SAA significantly decreased ADP-induced platelet aggregation, eliciting an inhibitory effect similar to Tirofiban. Therefore, SAA may reduce coronary thrombus by inhibiting platelet activation and improving coronary blood flow.

The inflammatory response and platelet aggregation have been implicated in myocardial I/R injury (19). Within min of reperfusion, an inflammatory cascade is triggered and numerous pro-inflammatory cytokines are released into the region, including TNF- $\alpha$, IL- $1 \beta$, IL-6 and IL-8. These pro-inflammatory cytokines, particularly TNF- $\alpha$, function as key mediators in cardiac dysfunction, serving to activate endothelial cells and neutrophils and aggravate myocardial I/R injury (20). In addition to regulating thrombosis, SAA modulates the inflammatory process (21). During adhesion, platelets are activated and release a variety of potent chemotactic factors, as previously described (22). Moreover, platelets may modulate the chemotactic properties of other cells through platelet-leukocyte (23) and platelet-endothelium interactions (24).

$\mathrm{P}$-selectin is present on activated platelets and mediates loose contact between circulating platelets and the vascular endothelium. Once platelets are activated during ischemia and reperfusion, p-selectin may be cleaved from the membrane to generate a soluble form that is readily detected in the plasma (25). The present study observed that levels of p-selectin were significantly elevated in the myocardium after 3 and $24 \mathrm{~h}$ of reperfusion when compared to the sham group, indicating that platelet activation occurs as a result of I/R. Furthermore, it was observed that SAA decreased levels of serum p-selectin and plasma IL-1 $\beta$ at 3 and $24 \mathrm{~h}$ after reperfusion, and reduced plasma levels of TNF- $\alpha$ at $24 \mathrm{~h}$ after reperfusion. Reduced cytokine levels are both a cause and consequence of reduced inflammatory cell infiltration. These data suggest that SAA functions to reduce platelet activation and inflammation.

NO is a diatomic free-radical gas that serves key roles in a numerous of biological processes, including inhibited platelet aggregation via a cyclic GMP-dependent mechanism and relaxed vascular endothelium as an endothelium derived relaxation factor (16). Maintaining a basal level of NO is critical for various physiological processes, including vascular smooth muscle cell relaxation, prevention of neutrophil and platelet adhesion to the endothelium, and maintenance of an anti-apoptotic environment 
in the vessel wall. I/R alters the levels of NO to further aggravate I/R injury (26). Furthermore, it has been documented that Tirofiban increased NO production, and that SAA alleviated impaired expression of endothelial nitric oxide synthase and NO formation in response to I/R $(10,27)$. Similarly, results of the present study indicated that SAA protected endothelial cells by improving NO production within the vessel wall.

SAA has been demonstrated to prevent I/R-induced myocardial damage by reducing cardiomyocyte necrosis and apoptosis $(28,29)$. The present study revealed that SAA and Tirofiban share similarities in terms of inhibiting the ADP-induced platelet aggregation rate, reducing plasma levels of p-selectin, reducing inflammatory factors and increasing the generation of NO to reduce myocardial infarct size. These similarities suggest that SAA may protect myocardial cells principally through antiplatelet activity. Notably, Tirofiban does not increase the survival rate of rats following exposure to I/R, potentially due to severe hemorrhage complications associated with its use (30-32).

In conclusion, the present study demonstrated that SAA protected the myocardium against I/R injury and improved survival rate by reducing platelet activation and inflammation. The protective effects of SAA may be principally related to the antiplatelet effects of SAA. Thus, SAA may be viable as a novel antiplatelet drug for use in both ischemic heart disease and cardiac surgeries associated with I/R injury.

\section{Acknowledgements}

The present study was supported by the Technology Bureau of Lishui (grant no. 20110410).

\section{References}

1. Moran AE, Forouzanfar MH, Roth GA, Mensah GA, Ezzati M, Flaxman A, Murray CJ and Naghavi M: The global burden of ischemic heart disease in 1990 and 2010: The Global Burden of Disease 2010 study. Circulation 129: 1493-1501, 2014.

2. Fröhlich GM, Meier P, White SK, Yellon DM and Hausenloy DJ: Myocardial reperfusion injury: Looking beyond primary PCI. Eur Heart J 34: 1714-1722, 2013.

3. Becker LC and Ambrosio G: Myocardial consequences of reperfusion. Prog Cardiovasc Dis 30: 23-44, 1987.

4. Maxwell SR and Lip GY: Reperfusion injury: A review of the pathophysiology, clinical manifestations and therapeutic options. Int J Cardiol 58: 95-117, 1997.

5. Piper HM, Meuter K and Schäfer C: Cellular mechanisms of ischemia-reperfusion injury. Ann Thorac Surg 75: S644-S648, 2003.

6. Pachel C, Mathes D, Arias-Loza AP, Heitzmann W, Nordbeck P, Deppermann C, Lorenz V, Hofmann U, Nieswandt B and Frantz S: Inhibition of platelet GPVI protects against myocardial ischemia-reperfusion injury. Arterioscler Thromb Vasc Biol 36: 629-635, 2016.

7. Gawaz M, Langer $\mathrm{H}$ and May AE: Platelets in inflammation and atherogenesis. J Clin Invest 115: 3378-3384, 2005.

8. Klinger MH and Jelkmann W: Role of blood platelets in infection and inflammation. J Interferon Cytokine Res 22: 913-922, 2002.

9. Skyschally A, Erbel R and Heusch G: Coronary microembolization Circ J 67: 279-286, 2003

10. Liu X and Tao GZ: Effects of tirofiban on the reperfusion-related no-reflow in rats with acute myocardial infarction. J Geriatr Cardiol 10: 52-58, 2013.

11. Howard JP, Jones DA, Gallagher S, Rathod K, Antoniou S, Wright P, Knight C, Mathur A, Weerackody R and Wragg A: Glycoprotein $\mathrm{IIb} / \mathrm{III}$ inhibitors use and outcome after percutaneous coronary intervention for non-ST elevation myocardial infarction. Biomed Res Int 2014: 643981, 2014.

12. Cheng TO: Cardiovascular effects of Danshen. Int J Cardiol 121 9-22, 2007.
13. Han JY,Fan JY,Horie Y, Miura S, Cui DH, Ishii H,Hibi T, Tsuneki H and Kimura I: Ameliorating effects of compounds derived from Salvia miltiorrhiza root extract on microcirculatory disturbance and target organ injury by ischemia and reperfusion. Pharmacol Ther 117: 280-295, 2008.

14. Lian-Niang L, Rui T and Wei-Ming C: Salvianolic acid A, a new depside from roots of Salvia miltiorrhiza. Planta Med 50: 227-228, 1984.

15. Huang ZS, Zeng CL, Zhu LJ, Jiang L, Li N and Hu H: Salvianolic acid A inhibits platelet activation and arterial thrombosis via inhibition of phosphoinositide 3-kinase. J Thromb Haemost 8: 1383-1393, 2010.

16. Moncada S, Palmer RM and Higgs EA: Nitric oxide: Physiology, pathophysiology, and pharmacology. Pharmacol Rev 43: 109-142, 1991.

17. Liu X and Tao GZ: Effects of tirofiban on the reperfusion-related no-reflow in rats with acute myocardial infarction. J Geriatr Cardiol 10: 52-58, 2013.

18. Ruggeri ZM: Platelets in atherothrombosis. Nat Med 8: 1227-1234, 2002.

19. Hansen PR: Inflammatory alterations in the myocardial microcirculation. J Mol Cell Cardiol 30: 2555-2559, 1998.

20. Vinten-Johansen J, Jiang R, Reeves JG, Mykytenko J, Deneve J and Jobe LJ: Inflammation, proinflammatory mediators and myocardial ischemia-reperfusion injury. Hematol Oncol Clin North Am 21: $123-145,2007$.

21. Li J, Gu T, Fu X and Zhao R: Effect of salvianolic acid A and C compatibility on inflammatory cytokines in rats with unilateral ureteral obstruction. J Tradit Chin Med 35: 564-70, 2015

22. Rendu F and Brohard-Bohn B: The platelet release reaction: Granules' constituents, secretion and functions. Platelets 12: 261-273, 2001.

23. Neumann FJ, Marx N, Gawaz M, Brand K, Ott I, Rokitta C, Sticherling C, Meinl C, May A and Schömig A: Induction of cytokine expression in leukocytes by binding of thrombin-stimulated platelets. Circulation 95: 2387-2394, 1997.

24. Gawaz M, Neumann FJ, Dickfeld T, Koch W, Laugwitz KL, Adelsberger H, Langenbrink K, Page S, Neumeier D, Schömig A and Brand K: Activated platelets induce monocyte chemotactic protein-1 secretion and surface expression of intercellular adhesion molecule-1 on endothelial cells. Circulation 98: 1164-1171, 1998.

25. Massberg S, Enders G, Leiderer R, Eisenmenger S, Vestweber D, Krombach F and Messmer K: Platelet-endothelial cell interactions during ischemia/reperfusion: The role of P-selectin. Blood 92: 507-515, 1998 .

26. Rafikov R, Fonseca FV, Kumar S, Pardo D, Darragh C, Elms S, Fulton D and Black SM: eNOS activation and NO function: Structural motifs responsible for the posttranslational control of endothelial nitric oxide synthase activity. J Endocrinol 210: 271-284, 2011.

27. Yang D, Xie P and Liu Z: Ischemia/reperfusion-induced MKP-3 impairs endothelial NO formation via inactivation of ERK1/2 pathway. PLoS One 7: e42076, 2012.

28. Pan H, Li D, Fang F, Chen D, Qi L, Zhang R, Xu T and Sun H: Salvianolic acid A demonstrates cardioprotective effects in rat hearts and cardiomyocytes after ischemia/reperfusion injury. J Cardiovasc Pharmacol 58: 535-542, 2011.

29. Fan H, Yang L, Fu F, Xu H, Meng Q, Zhu H, Teng L, Yang M, Zhang L, Zhang Z and Liu K: Cardioprotective effects of salvianolic acid $\mathrm{A}$ on myocardial ischemia-reperfusion injury in vivo and in vitro. Evid Based Complement Alternat Med 2012: 508938, 2012.

30. Aguirre FV, Topol EJ, Ferguson JJ, Anderson K, Blankenship JC, Heuser RR, Sigmon K, Taylor M, Gottlieb R, Hanovich G, et al: Bleeding complications with the chimeric antibody to platelet glycoprotein IIb/IIIa integrin in patients undergoing percutaneous coronary intervention. EPIC Investigators. Circulation 91: 2882-2890, 1995

31. Kellert L, Hametner C, Rohde S, Bendszus M, Hacke W, Ringleb P and Stampfl S: Endovascular stroke therapy: Tirofiban is associated with risk of fatal intracerebral hemorrhage and poor outcome. Stroke 44: 1453-1455, 2013.

32. Ilhan E, Güvenc TS, Güzelburc O, Altay S, Özer N, Soylu O, Hasdemir $\mathrm{H}$ and Ergelen M: A fatal complication of tirofiban in an octogenarian: Diffuse alveolar hemorrhage. J Cardiol Cases 2: e48-e51, 2010. 